\title{
Reproductive Health and Fertility in Transition Clinic for Liver Transplant Patients
}

\author{
Uyen $^{1 *}$, Alla Vash-Margita ${ }^{2}$ and Michael Schilsky ${ }^{1,2}$ \\ ${ }^{1}$ Assistant Professor of Disease, Department of Digestive Disease and Transplant, USA \\ ${ }^{2}$ Assistant Professor, Department of Obstetrics, Gynecology and Reproductive Sciences, Chief, Pediatric and Adolescent Gynecology, USA \\ *Corresponding author: Uyen MD, Assistant Professor of Disease, Department of Digestive Disease and Transplant, Yale University, \\ USA
}

\section{Short Communication}

The transition of an adolescent into adulthood is fraught with complexity under the best of circumstances. Misinformation or a lack of medical knowledge during this critical time can place young adults in a position to develop risky behaviors. However, simple well-timed educational interventions can foster healthy, informed decisions which can last a lifetime. Adolescents who have undergone pediatric liver transplantation are a subgroup of teenagers at a particularly elevated risk, in part due to their immunosuppression and comorbid conditions, during this time of developing personal autonomy. As these patients begin to develop and establish their personal and reproductive identities, they may also have limited understanding of the long-term consequences of their decisions about reproductive health, contraception, and sexual activity. A national survey of adult transplant hepatologists showed that only $46 \%$ of patients who were transitioned from pediatric to adult care understood their underlying condition [1]. Furthermore, only $15 \%$ of transplant programs have a formal transition clinic [1]. The establishment of a transition clinic has been shown to improve medication adherence, laboratory testing and visit follow up. It also allows for patients to be able to understand the impact of their condition on their life including their sexual and reproductive health $[1,2]$. One of the barriers that adult hepatologists encounter in transitioning patients from pediatric to adult practice is a lack of training in adolescent sexual and reproductive health. Incorporating a gynecologist with expertise in adolescent health into the transition clinic can be helpful to address this deficit.

Female patients with end stage liver disease are often infertile in the setting of metabolic, nutritional and endocrine derangements. Issues with the hypothalamic pituitary axis and estrogen metabolism have led to issues of amenorrhea and anovulation [3]. However, following liver transplant, approximately $80 \%$ of patients will have return of their menstrual cycle and their fertility within 8-12 months [4]. In a telephone survey, 49\% $(n=34)$ of women within child bearing age with a liver transplant were not aware that they could become pregnant after transplant [4]. In the same study, they also noted that only $37 \%(n=67)$ of women following solid organ transplant (kidney, liver, intestine or pancreas) had discussions with their providers regarding methods of contraception [4]. Education regarding fertility and contraception is especially important for liver transplant patients, including a thorough discussion of what to expect should they become pregnant, and a discussion of the potential complications. Pregnancy in liver transplant patients can be complicated by preterm labor, graft rejection and loss, increased risk of pre-eclampsia, and an increased risk of infections and diabetes in the mother among others [5]. In terms of the fetus, prematurity and low birth weight can occur [5]. In addition, immunosuppression doses and regimens often require adjustment when a liver transplant patient is planning to become pregnant. In a study of immunosuppression in our pediatric patients transitioning from adolescence to adulthood in 2005, 92\% of patients were on tacrolimus [6]. There was a drop in the use of azathioprine and a rise of the use of mycophenolate mofetil from 3.9\% to 28\% during this time period [6]. A lack of understanding of the side-effects of immune suppression is unfortunately common amongst our patients transitioning from adolescence to adulthood [7].

For example, many do not know that mycophenolate mofetil can cause first term trimester miscarriage and fetal malformations and should be avoided 6 weeks before a planned conception [8]. Contraception can also be important in women of reproductive age who undergo liver transplant, as pregnancy in the first 1-2 years 
after transplant carries an increased burden of complications [5]. The most common contraception methods discussed with patients are barrier methods such as condoms, followed by oral contraceptives, and less commonly intrauterine devices [9]. In one study of kidney and liver transplant patient recipients, almost half of patients were using no contraception and approximately $40 \%$ of women were relying on high failure methods such as condoms, withdrawal, or rhythm [9]. In another survey study, kidney or liver transplant patients reported not receiving any counseling by obstetricians/gynecologists and would have liked to have more counseling on more highly effective contraceptive methods [10]. They also indicated preference for contraceptive counseling to occur before transplant and to have it available in the transplant clinic [10]. Long term use of immunosuppression has also been linked to an elevated risk of cervical cancer and thus patients should be engaged with gynecologist early on for routine screening [11]. The 2- to 3-dose regimen of 9-valent HPV vaccine is recommended for immunosuppressed adolescents [12]. Efficacy of quadrivalent HPV vaccine is decreased in patients with organ transplant [13]. There are no comparable studies for the vaccine that targets 9 types of HPV virus. The age limit for the use of 9-valent vaccine was extended by the FDA in October of 2018 and is now approved for use in women and men aged 27 to 45 years. Cervical cancer screening is recommended for immunocompetent females starting at age 21 [14]. Young women following solid organ transplant or immunocompromise from any source should start cervical cancer screening within 1 year of onset of sexual activity but no later that 21 years of age. There are no separate guidelines for cervical cancer screening in women following solid organ transplant. Instead, initiation, frequency and management of abnormal cytologic findings is followed according to the guidelines developed for women with HIV [15].

There are also no specific guidelines for sexually transmitted infections (STI) screening in women with solid organ transplant or for those immunocompromised by other causes except for women with HIV. For women with HIV the American College of Obstetricians and Gynecologists recommends screening for Neisseria gonorrhoeae and Chlamydia trachomatis with a nucleic acid amplification test, serologic testing for syphilis, and testing for vaginal trichomoniasis at entry of care and annually thereafter [16]. It is unclear whether these guidelines can be extrapolated to recipients of solid organ transplant [17]. Screening for sexually transmitted diseases remains underutilized in recipients of solid organ transplant. In a study by Ashoor IF et al. [18], only $43 \%$ of providers routinely or always asked about sexual activity [18]. Universal screening for STI's at predefined intervals was performed by only $10 \%$ of providers for adolescent kidney transplant recipients. Major obstacles for universal screening for STI's are lack of training in this area by the transplant providers, absence of protocols for STI evaluation and fear of breach of confidentiality, especially in adolescent patients. There are many facets to the complex care of the adolescent liver transplant patient transitioning into adulthood. We highlight here the often-missed opportunities to illuminate how reproductive health issues can intersect with adolescents in the post-transplant state, during a critical time of their personal and sexual development, when they are at significant risk of unplanned pregnancy or transmission of infections. The addition of a gynecologist with subspecialty training in pediatric and adolescent gynecology to the transition transplant clinic could help increase responsible awareness of these issues in our population.

\section{References}

1. Heldman MR (2015) National survey of adult transplant hepatologists on the pediatric-to-adult care transition after liver transplantation. Liver Transpl 21(2): 213-223.

2. Mitchell T (2017) Transition to adult care for pediatric liver transplant recipients: The Western Australian experience. Pediatr Transplant 21(1).

3. Jabiry-Zieniewicz Z (2009) Menstrual function in female liver transplant recipients of reproductive age. Transplant Proc 41(5): 1735-1739.

4. French VA (2013) Contraception and fertility awareness among women with solid organ transplants. Obstet Gynecol 122(4): 809-814.

5. Sarkar M (2018) Reproductive health in women following abdominal organ transplant. Am J Transplant 18(5): 1068-1076.

6. Horslen S (2007) Pediatric transplantation in the United States, 19962005. Am J Transplant 7(5 Pt 2): 1339-1358.

7. Shemesh E (2004) Medication Adherence in Pediatric and Adolescent Liver Transplant Recipients. Pediatrics 113(4): 825-832.

8. Hoeltzenbein M (2012) Teratogenicity of mycophenolate confirmed in a prospective study of the European Network of Teratology Information Services. Am J Med Genet A 158A (3): 588-96.

9. Szymusik I (2014) Contraception in women after organ transplantation. Transplant Proc 46(10): 3268-3272.

10. Rafie S (2014) Contraceptive use in female recipients of a solid-organ transplant. Prog Transplant 24(4): 344-348.

11. Madeleine MM (2013) HPV-related cancers after solid organ transplantation in the United States. Am J Transplant 13(12): $3202-$ 3209.

12. (2017) Committee Opinion No. 704: Human Papillomavirus Vaccination. Obstet Gynecol 129(6): e173-e178.

13. Kumar D (2013) Immunogenicity of quadrivalent human papillomavirus vaccine in organ transplant recipients. Am J Transplant 13(9): 24112417.

14. (2016) Practice Bulletin No. 168: Cervical Cancer Screening and Prevention. Obstet Gynecol 128(4): e111-130.

15. (2016) Practice Bulletin No. 167: Gynecologic Care for Women and Adolescents With Human Immunodeficiency Virus. Obstet Gynecol 128(4): e89-e110

16. (2016) Practice Bulletin No. 167 Summary: Gynecologic Care for Women and Adolescents With Human Immunodeficiency Virus. Obstet Gynecol 128(4): 920-922.

17. Krajewski C, G Sucato (2014) Reproductive health care after transplantation. Best Pract Res Clin Obstet Gynaecol 28(8): 1222-1234.

18. Ashoor IF, VR Dharnidharka (2015) Sexually transmitted infection screening and reproductive health counseling in adolescent renal transplant recipients: Perceptions and practice patterns. A study from the Midwest Pediatric Nephrology Consortium. Pediatr Transplant 19(7): 704-708. 
(C) This work is licensed under Creative

To Submit Your Article Click Here:

Submit Article

DOI: $10.32474 /$ CTGH.2019.02.000126

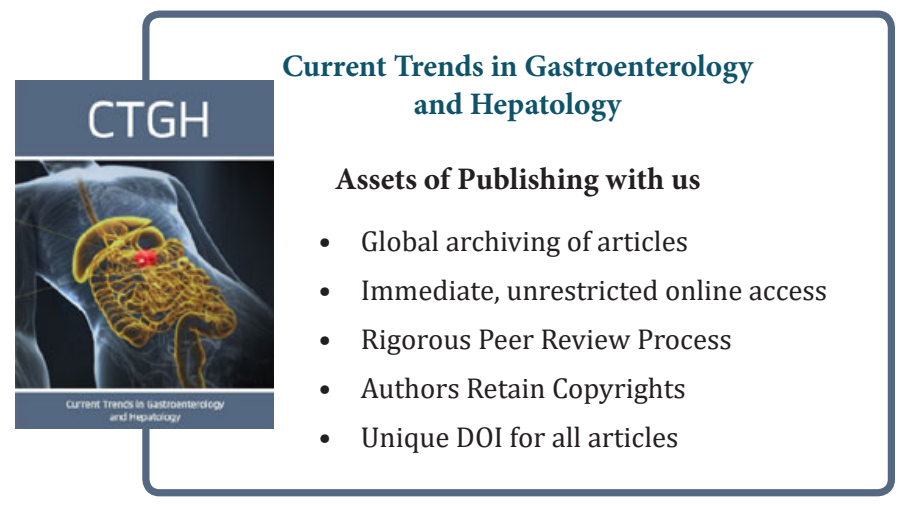

